\section{(1)}

CrossMark

\title{
Early consensus management for non- ICU acute respiratory failure SARS-CoV-2 emergency in Italy: from ward to trenches
}

\author{
Michele Vitacca (10 ${ }^{1}$, Stefano Nava ${ }^{2,3}$, Pierachille Santus ${ }^{4}$ and Sergio Harari $\mathbb{1}^{5}$ \\ Affiliations: ${ }^{1}$ ICS Maugeri IRCCS, Respiratory Rehabilitation Unit Lumezzane, Brescia, Italy. ${ }^{2}$ Alma Mater \\ Studiorum University of Bologna, Dept of Clinical, Integrated and Experimental Medicine (DIMES), Bologna, \\ Italy. ${ }^{3}$ Respiratory and Critical Care, Sant'Orsola Malpighi Hospital, Bologna, Italy. ${ }^{4}$ Dept of Biomedical and \\ Clinical Sciences "L. Sacco", Università degli Studi di Milano. Division of Respiratory Diseases, L. Sacco \\ University Hospital - ASST Fatebenefratelli Sacco, Milan, Italy. ${ }^{5}$ Dept of Medical Sciences, San Giuseppe \\ Hospital MultiMedica IRCCS, and Department of Clinical Sciences and Community Health, \\ UniversitàdegliStudi di Milano, Milan, Italy.
}

Correspondence: Sergio Harari, Dept of Medical Sciences, San Giuseppe Hospital MultiMedica IRCCS, and Department of Clinical Sciences and Community Health, UniversitàdegliStudi di Milano, Via San, Vittore 12 , 20123 Milan, Italy. E-mail: sergiodsergioharari.it

@ERSpublications

The development, as soon as possible, of a European respiratory specialist network is mandatory to manage the unexpected emergency of SARS-CoV-2; the ERS has a key role to play in urgently providing recommendations, guidelines, support and information https://bit.ly/39OaH0o

Cite this article as: Vitacca M, Nava S, Santus P, et al. Early consensus management for non-ICU acute respiratory failure SARS-CoV-2 emergency in Italy: from ward to trenches. Eur Respir J 2020; 55: 2000632 [https://doi.org/10.1183/13993003.00632-2020].

The number of people infected with severe acute respiratory syndrome coronavirus 2 (SARS-CoV-2) is increasing dramatically throughout the world [1], and in Italy [2,3], particularly in the northern region of Lombardy [4]. Regional Italian medical and political authorities have implemented extraordinary measures to contain the spread of the virus. This disease can cause massive diffuse alveolar damage resulting in acute respiratory failure (ARF), which requires, in a high percentage of cases, mechanical ventilation [5-8]. Based on our general experience so far in dealing with the disease and on the existing knowledge (albeit still limited and evolving) [5-8], Italian respiratory scientific societies herein propose early consensus statement management for non-intensive care unit (ICU) ARF SARS-CoV-2 emergencies. The consensus statement represents the expert opinion of pulmonologists directly involved in the first line of assistance, and has identified two urgent areas of action: management and organisation.

We carried out a search of the published literature in PubMed, Ovid, Embase databases and relevant websites, with the searches ranging from the construction of the databases to 20 March 2020 in order to retrieve guidelines and recommendations, meta-analyses, systematic reviews, state-of-the-art papers and randomised trials. The search terms used were: "coronavirus pneumonia", "ARDS", "acute hypoxemic respiratory failure", "SARS", "MERS”, "influenza", "acute respiratory failure or mechanical ventilation", and "noninvasive ventilation AND acute hypoxemic respiratory failure". Based on the literature search, a small group of four pulmonologists produced a preliminary document, which was then submitted to a consensus 
group of 10 doctors. Consensus on the final document was achieved through video conference meetings. Using a Delphi-like procedure, we asked the experts to rate the entire document on a 5-point Likert scale ( 0 =totally disagree; $1=$ disagree; $2=$ sufficiently agree; $3=$ moderately agree; $4=$ totally agree). Consensus was considered when $>75 \%$ of the respondents rated the document as "totally agree". In this context, the proposed paper is a changeable consensus, which is not necessarily totally in line with the World Health Organization (WHO) documents because the Italian situation was and unfortunately still is continuously in progression. Below, we summarise the recommendations that we consider most appropriate and urgent.

The management-related actions regarded, first, the need to ensure maximum protection of doctors and nurses working in the field (e.g. working at a distance of $\geqslant 1 \mathrm{~m}$ from the suspected or positive patient). Second, the need to create an epidemiological/clinical assessment protocol (triage) to classify patients based on medical history, geographical origin (i.e. inside or outside the cluster zones) and clinical signs such as fever, persistent cough for $>48-72 \mathrm{~h}$, dyspnoea and arterial oxygen saturation $\left(S_{\mathrm{aO}_{2}}\right)<93 \%$ when breathing air. Third, the need to develop a diagnostic algorithm to determine which tests to perform and when, i.e. pharyngeal swab for SARS-CoV-2, chest radiography or pulmonary high-resolution computed tomography (CT).

The proposed triage led to the identification of four patient categories: green $\left(S_{\mathrm{aO}_{2}}>94 \%\right.$, respiratory rate (RR) $<20$ breaths. $\left.\mathrm{min}^{-1}\right)$; yellow $\left(S_{\mathrm{aO}_{2}}<94 \%, \mathrm{RR}>20\right.$ breaths $\cdot \mathrm{min}^{-1}$ but responds to oxygen $10-$ $\left.15 \mathrm{~L} \cdot \mathrm{min}^{-1}\right)$; orange $\left(S_{\mathrm{aO}_{2}}<94 \%\right.$, RR $25-30$ breaths $\cdot \mathrm{min}^{-1}$ but poor response to oxygen $10-15 \mathrm{~L} \cdot \mathrm{min}^{-1}$ and requires continuous positive airway pressure (CPAP)/noninvasive ventilation (NIV) with inspiratory oxygen fraction $\left(F_{\mathrm{IO}_{2}}\right)<50 \%$; red $\left(S_{\mathrm{aO}_{2}}<94 \%\right.$, RR $>30$ breaths $\mathrm{min}^{-1}$ but poor response to oxygen 10 $15 \mathrm{~L} \cdot \mathrm{min}^{-1}$, CPAP/NIV with $\mathrm{F}_{\mathrm{IO}_{2}}>50 \%$ or presenting respiratory distress with arterial oxygen tension $\left(P_{\mathrm{aO}_{2}}\right) / F_{\mathrm{IO}_{2}}<200$ and needing endotracheal intubation (EI) and ICU admission).

The actions requested concerned indications for the transfer of suspected or confirmed cases, depending on the local situation, to one of the following: 1) ad hoc so-called "COVID Hub" hospitals (i.e. special units inside or outside the hospital dedicated to these patients and developed, on average, within or shortly after the first week of the initial outbreak); 2) infectious disease units, or dedicated areas ready for isolation of confirmed cases and immediate ARF treatment; or 3) ICU for early intubation of compromised patients with low $P_{\mathrm{aO}_{2}} / F_{\mathrm{IO}_{2}}$ or patients "not responding" to oxygen/CPAP/NIV.

The consensus group focused on a "what to do" management pathway (figure 1), stressing the need for close patient monitoring, care of comorbidities, fluid and nutritional prescription, sedation if needed, use of aerosol devices if needed, and monitoring of the risk of a sudden deterioration in the patient's clinical condition. It discussed the indications for high-flow oxygen blender, the level of $F_{\mathrm{IO}_{2}}$ to guarantee an $S_{\mathrm{aO}_{2}}$ of $>90 \%$, high-flow nasal cannula oxygen devices, and CPAP/NIV indications as a form of treatment or as ceiling of treatment and palliative care.

After extensive discussion, the consensus set the indication for CPAP use at $10-12 \mathrm{cmH}_{2} \mathrm{O}$, without humidification and with helmet (first choice), for CPAP use with a mask (second choice) and for NIV use with an oronasal face mask (third choice), using high performance ventilators or, if these are lacking, dedicated NIV platforms or home ventilators. Due to an insufficient number of ICU beds, respiratory intermediate units and negative pressure rooms able to provide respiratory support to all patients, the consensus group proposed that emergency rooms, medical wards, dedicated units and surgical rooms should be transformed into locations that could provide any form of mechanical ventilation, in rooms with the possibility of air exchange (big windows opened periodically, achieving a change of air of $\geqslant 160 \mathrm{~L} \cdot \mathrm{h}^{-1}$, as recommended). Clearly, when all of these environments are unavailable, CPAP/NIV is indicated using the maximum available personnel protection.

Indications for when to stop CPAP/NIV in case of worsening did not reach a consensus amongst the audit group, nor did standardised indications for when to perform intubation, as this depends on the number of beds of the referral ICU and their actual occupancy.

Obviously, the group suggested regular decontamination of the ventilator and devices used, at the end of ventilator treatment. Due to uncertainty around the potential for aerosolisation, high-flow oxygen, NIV and bubble CPAP, we further stressed the need for airborne precautions. The consensus group also stressed that, in de novo severe critical patients and in patients failing $2 \mathrm{~h}$ of CPAP/NIV, the gold standard remains ICU admission. Otherwise, in "war" conditions like this with huge numbers of patients, and a paucity of ICU beds and ventilators, administering CPAP/NIV noinvasively remains an option.

The consensus group did not focus on the role of antivirals, antibiotics, steroids, anticoagulants and other innovative drugs because this topic was not strictly related to the consensus objective.

Regarding organisation, pulmonologists have been involved in identifying: 1) hospitals or hospital areas for isolated suspect patients awaiting confirmation of diagnosis; 2) specific "contaminated" paths, zones and 


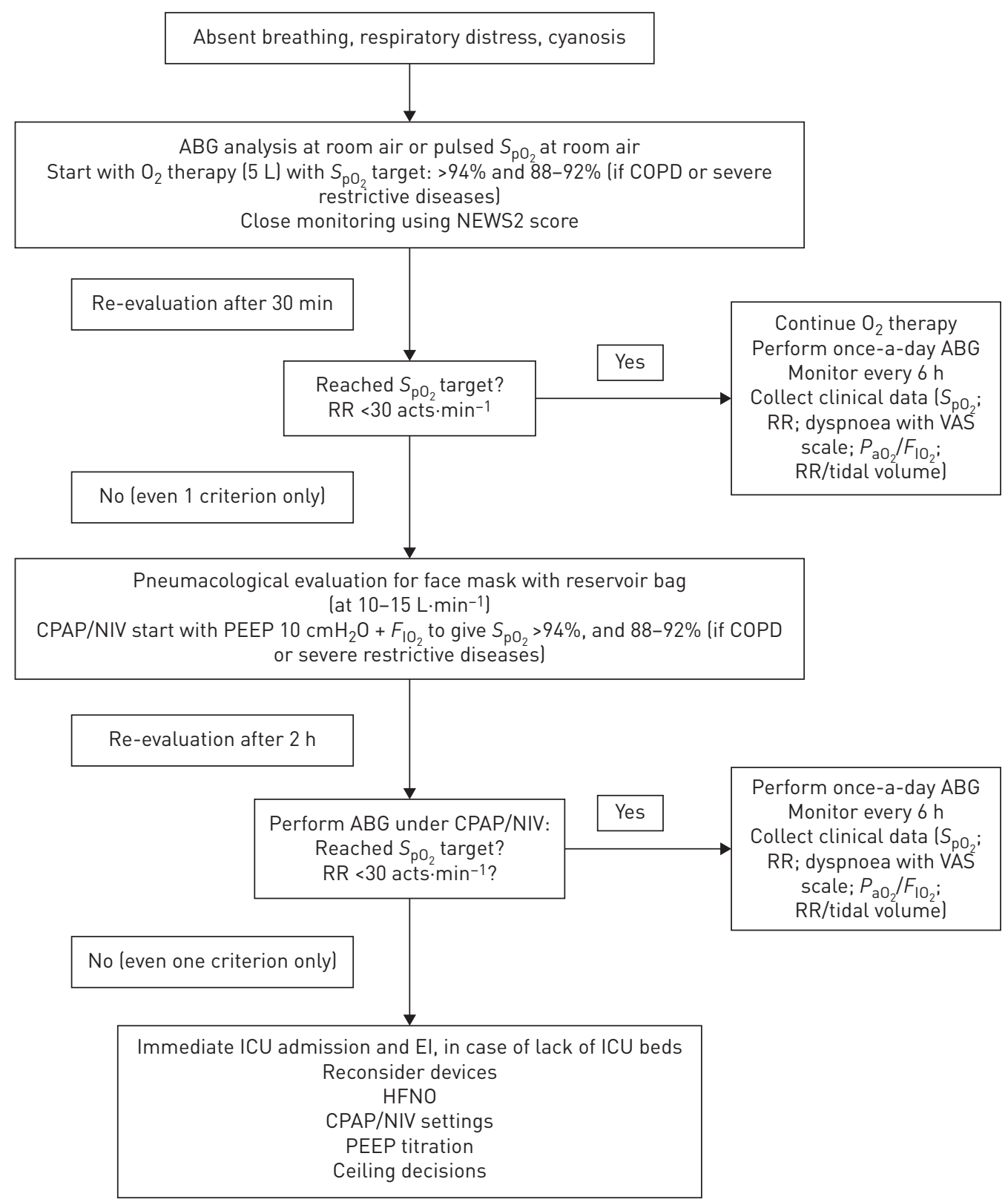

FIGURE 1 "What to do" management pathway. ABG: arterial blood gases; $\mathrm{S}_{\mathrm{pO}_{2}}$ : oxygen saturation measured by pulse oximetry; NEWS2: national early warning score; RR: respiratory rate; VAS: visual analogue scale; $P_{\mathrm{aO}}$ : arterial oxygen tension; $F_{\mathrm{IO}_{2}}$ : inspiratory oxygen fraction; CPAP: continuous positive airway pressure; NIV: noninvasive ventilation; PEEP: positive end-expiratory pressure; ICU: intensive care unit; EI: endotracheal intubation; HFNO: high-flow nasal oxygen.

the team involved; 3) transferal procedures for patients into negative aeration rooms (when available) or, as a second choice, into one-bed rooms or an area with a distance of $\geqslant 2 \mathrm{~m}$ between patients. The work led to the creation of isolation areas for patient groups (positive and on EI; positive and on NIV/CPAP; positive with respiratory failure on oxygen therapy; negative pending the second pharyngeal swab if the clinic and CT scan suggest bilateral and interstitial pneumonia). The respiratory team had to manage and co-manage patients with other specialists in the multidisciplinary team, with maximal flexibility to find discharge routes to "intermediate" units (such as internal medicine, respiratory rehabilitation, social units) for coronavirus 2019 (COVID-19) patients in further need of clinical and infective follow-up. The consensus group agreed to abolish visits by family members to COVID-19 patients, offering once-a-day face-to-face contact or a telephone call with one family member only.

Since the first days of the crisis, our teams have been involved in sharing ceiling treatment decisions regarding EI and CPAP/NIV use based on the patient's medical history and age, the beds available and the 


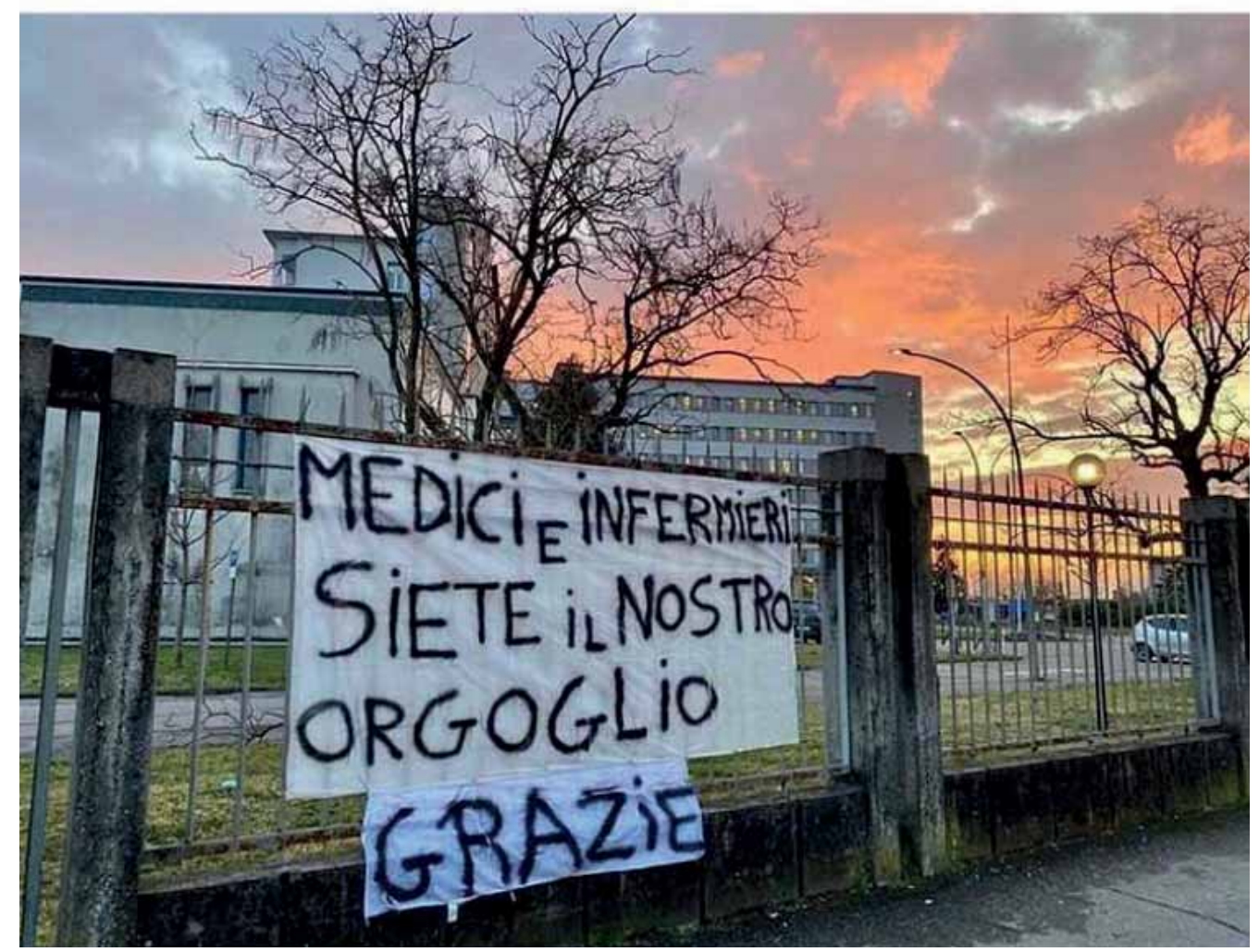

FIGURE 2 The Italian population understand the situation of healthcare staff ("Doctors and nurses, you are our pride, thank you").

number of new cases. All our staff has been strongly exhorted to use adequate personal protective equipment according to the WHO document [9], equipment that is still difficult to find due to the huge demand.

As of 28 March 2020, in Italy, $>86000$ people have been found to be COVID-19 positive, 40000 have been isolated at home, 4000 have been admitted to ICU, 6000 have been ventilated noninvasively, 9000 died and 11000 have recovered. A large number of patients are co-managed by mixed teams of pulmonologists and others specialists. Our respiratory teams are living in a constant state of anxiety, anguish, fear, helplessness, panic, despondency and inadequacy, with moments of deafening silence and moments of excited frenzy, but at the same time with courage, firmness, determination, professionalism, solidarity and compassion. As illustrated in figure 2, the general public seems to be well aware and empathises with our particular situation.

In conclusion, based on our actual experience and the recent literature, it seems clear that the viral spread is destined to continue growing; hence, further extraordinary organisational proposals, detailed protocols and specialised teams are urgently required. We do not know if we are facing something similar to what was experienced by heroic doctors and nurses in the 1950s during the polio epidemic when most hospitals had limited availability of iron lungs for patients unable to breathe independently [10]. During that time, makeshift respiratory centres were set up to assist the most severe patients, and it was from this that ICU was born [10]. Our suggestions to our colleagues and health policy makers are: 1) develop clear and effective measures to protect healthcare workers as soon as possible; 2) immediately increase and maintain the beds in ICU and those dedicated to NIV and critical care patients in the respiratory setting; 3 ) completely rethink the different European health systems, considering a wide range of flexibility and the strong need for the development of homecare assistance and new models of hospital organisation; and 4) develop a "Marshall plan" strong enough to withstand the devastating impact the COVID-19 pandemic will have on disability and socioeconomic systems.

Acknowledgements: We thank all pulmonologists, nurses and healthcare workers involved in this dramatic emergency for their tireless dedication and availability. 
Conflict of interest: M. Vitacca has nothing to disclose. S. Nava has nothing to disclose. P. Santus has nothing to disclose. S. Harari reports grants and personal fees for lectures and membership of scientific advisory boards from Roche, outside the submitted work.

\section{References}

1 WHO. Global surveillance for human infection with coronavirus disease (COVID-2019). https://www.who.int/ publications-detail/global-surveillance-for-human-infection-with-novel-coronavirus-(2019-ncov) Date last accessed: 4 March 2020.

2 Istituto Superiore di Sanità. http://www.epicentro.iss.it/coronavirus/ Date last accessed: 4 March 2020.

3 European Centre for Disease Prevention and Control. https://www.ecdc.europa.eu/en/covid-19-pandemic Date last accessed: 4 March 2020.

4 Spina S, Marrazzo F, Migliari M, et al. The response of Milan's Emergency Medical System to the COVID-19 outbreak in Italy. Lancet 2020; 395: e49-e50.

5 Zhang Y, Xu J, Li H, et al. A novel coronavirus (COVID-19) outbreak: a call for action. Chest 2020; 157: e99-e101.

6 Zhu N, Zhang D, Wang W, et al. A novel coronavirus from patients with pneumonia in China, 2019. $N$ Engl J Med 2020; 382: 727-733.

7 Huang C, Wang Y, Li X, et al. Clinical features of patients infected with 2019 novel coronavirus in Wuhan, China. Lancet 2020; 395: 497-506.

$8 \mathrm{Wu}$ Z, McGoogan JM. Characteristics of and important lessons from the coronavirus disease 2019 (COVID-19) outbreak in China. JAMA 2020; 2019: 25-28.

9 World Health Organization. Rational use of personal protective equipment for coronavirus disease 2019 (COVID19). https://apps.who.int/iris/bitstream/handle/10665/331215/WHO-2019-nCov-IPCPPE_use-2020.1-eng.pdf Date last updated: 27 February 2020.

10 Pincock S. BjørnAage Ibsen. Lancet 2007; 370: 1538. 